\title{
EI discurso pseudopolítico de la segunda pantalla. \#EIDebateEnRTVE visto a través de sus prosumers
}

\author{
The pseudo-political discourse of the second screen. \\ \#ElDebateEnRTVE seen through its prosumers
}

Paz Villar-Hernández. Universidad de Valencia. España.

paz.villar@uv.es

$[\mathrm{CV}] \odot \mathrm{G}$

Este artículo ha sido elaborado en el marco del proyecto de investigación: PRODISNET-02. Procesos discursivos en Internet: Desplazamientos enunciativos y efectos hiperbólicos en el discurso político. RTI2018093523-B-100, del Ministerio de Ciencia, Innovación y Universidades.

Cómo citar este artículo / Referencia normalizada

Villar-Hernández, P. (2020). El discurso pseudopolítico de la segunda pantalla. \#ElDebateEnRTVE visto a través de sus prosumers. Revista Latina de Comunicación Social, (76), 121-141. https://www.doi.org/10.4185/RLCS-2020-1440

\begin{abstract}
RESUMEN
Introducción: Nuestro objeto de análisis es la conversación mantenida en Twitter en uno de los debates electorales de las elecciones generales del 28 de abril: el que tuvo lugar el 22 de abril en Televisión Española entre los candidatos de los cuatro principales partidos políticos: PP, PSOE, UP y Cs. Metodología: La metodología empleada en el trabajo es el análisis del discurso de base pragmática, en la que se emplean técnicas cuantitativas y cualitativas de análisis. Para este, se seleccionaron de forma aleatoria 1000 tuits de entre los más populares que habían empleado los hashtags promocionales del debate: \#EIDebateEnRTVE y \#ELDEBATEenRTVE. Resultados: Los resultados indican que los cuatro partidos analizados participaron en el debate paralelo generado en Twitter y que, si bien la mayor parte de las intervenciones en la segunda pantalla fueron de ataque, los partidos prefirieron sobre todo elogiar a su candidato, a excepción de Ciudadanos, que optó por el ataque al PSOE y luego por el elogio propio. Discusión y conclusiones: En cuanto a los temas tratados fueron no tanto políticos sino pseudopolíticos. Los memes, mensajes irónicos y bromas ocuparon un espacio reducido en esta conversación transmediática.
\end{abstract}

PALABRAS CLAVE: elecciones generales; 28 de abril; debate electoral; Twitter; análisis pragmático del discurso; pseudopolítica.

\footnotetext{
ABSTRACT

Introduction: Our object of analysis is the conversation held on Twitter in one of the election debates in the campaign in the Spanish General Elections of April 28th, 2019; precisely, the one that took place on April 22 on the public broadcasting corporation Radiotelevisión Española among the
} 
leaders of the four main political parties: the ruling Socialists, the conservative People's Party (PP), the centre-right Ciudadanos and the left-wing Unidas-Podemos. Methodology: The methodology used in the work is the Pragmatic Discourse Analysis, and quantitative and qualitative analysis techniques are used. The data was coded and analyzed with Atlas.ti software. For the analysis, a total of 1000 tweets were randomly selected from among the most popular Twitter debate hashtags: \#EIDebateEnRTVE and \#ELDEBATEenRTVE. Results: The results indicate that the four parties participated in the second screen conversation generated on Twitter. The parties mostly preferred to praise their candidate, instead of attacking others that was the sample results. On the other part was Ciudadanos, who decided to the attack the PSOE and only afterwards to praise their candidate. Debate and Conclusions: As for the issues discussed by audiences, they were not exactly political but pseudo-political. The memes, ironic messages and jokes were not especially prominent in this transmedia debate.

KEYWORDS: Spanish general election; April $28^{\text {th }}$; electoral debate; Twitter; pragmatic discourse analysis; pseudo-politics.

\section{CONTENIDOS}

1. Introducción y marco teórico. 2. Método. 3. Resultados. 4. Discusión y conclusiones. 5. Referencias bibliográficas.

\section{Introducción y marco teórico}

El debate electoral televisado entre candidatos a la presidencia de un país es una tradición muy enraizada en lugares como Estados Unidos -donde surgió el modelo- Alemania o Francia, si bien hasta hace bien poco no lo era tanto en países como España (Cantavella-Blasco et al., 2008). En este país el primer debate televisado tuvo lugar un 24 de mayo de 1993 en los estudios de Antena 3. El presidente del Gobierno en esos momentos, Felipe González y el candidato del principal partido de la oposición, José María Aznar fueron los convocados. A este debate le siguió otro, días después (el 31 de mayo) en la otra gran cadena privada, Tele 5. Sin embargo, tras esos dos iniciales debates se hizo el silencio y tuvimos que esperar 15 años para vivir un nuevo enfrentamiento dialéctico entre candidatos a la Presidencia del Gobierno de España.

El año 2011 fue especialmente trascendente por dos cuestiones: por un lado, porque uno de esos debates incorporó a representantes de otras fuerzas políticas, iniciando una tendencia que se ha consolidado con los años y, por otra parte, porque el debate electoral vivió una transformación fruto de la influencia del debate paralelo en Twitter. Aunque será en los debates y campañas de 2015 y 2016 cuando esa relación se impulse y consolide, y la conversación de la segunda pantalla de inicio a la Social TV o la Televisión 2.0 (Ruiz del Olmo y Bustos Díaz, 2017). Así transcurrió el debate que aquí analizamos, el del 22 de abril de 2019, que congregó a los candidatos a la Presidencia del Gobierno del PSOE, PP, Cs y Unidas Podemos.

Son muchas las perspectivas desde las que se han analizado los debates electorales en España; debates que, a juicio de Fernández García (1999, p. 84) son más bien un conjunto de "estructuras no dialógicas" o una consecución de "discursos yuxtapuestos". Este autor, que también analizó los debates entre Rodríguez Zapatero y Mariano Rajoy (2008) (Fernández García, 2009) y el de 2011 entre Rubalcaba y Rajoy (Fernández García, 2009) es uno de los muchos que desde el punto de vista lingüístico han analizado este momento conversacional. Esta perspectiva cuenta con una amplia tradición en España que incluye desde los iniciales trabajos de Blas Arroyo respecto al primer debate de 1993 (1998, 1999, 2003) hasta los de contiendas dialécticas posteriores (Cabrejas-Peñuela, 2015; 
Cabrejas-Peñuelas, 2015; Cabrejas-Peñuelas y Díez-Prados, 2014; Cantavella-Blasco et al., 2008; Cuenca y Marín, 2015; Díez-Prados y Cabrejas-Peñuelas, 2018). Desde el ámbito comunicativo encontramos investigaciones que versan sobre sus aspectos formales, legislativos o de regulación (Rúas Araújo, Fernández Holgado, y Alén Amil, 2018), otros sobre la influencia del debate en la intención de voto (Callejón, 2001), su estructura narrativa audiovisual (Quintas Froufe y Quintas Froufe, 2010), la cobertura mediática hecha por medios tradicionales (García-Marín, 2015), la agenda temática de alguno de ellos (López-García, Llorca-Abad, Valera-Ordaz, y Peris-Blanes, 2018) y las sinergias entre el debate televisado y su comentario en la segunda pantalla (Ruiz del Olmo y Bustos Díaz, 2017).

Los estudios sobre el debate electoral comentado a través de redes sociales como Twitter cuentan, lógicamente, con una menor tradición (Vergeer y Franses, 2016). Esta práctica comunicativodiscursiva de generación de comentarios en redes sociales fruto de la visualización de programas televisivos, ha sido denominada por algunos como "viewertariat" (Anstead y O'Loughlin, 2011), o "sofalising" (Doughty, Rowland, y Lawson, 2012) y es ampliamente conocida como el fenómeno de la segunda o doble pantalla (Doughty et al., 2012; Elmer, 2012). En sí, ha cambiado radicalmente nuestra manera de ver la televisión. Ahora, las audiencias pueden evaluar en tiempo real lo que está ocurriendo en la televisión, actuando como "backchannel" (canal trasero) (Kalsnes, 2014; Pedersen et al., 2015) y aportando información segmentada y detallada sobre su respuesta a cada uno de los mensajes que los candidatos trasladan desde la pantalla (Vergeer y Franses, 2016), hasta el punto de que esta práctica: "is shaping the political realm across the world" (Gil de Zúñiga y Liu, 2017, p. 215).

Su alcance es evidente porque, pese a las diferentes motivaciones que guían su uso, según se trate de un país u otro (Gil de Zúñiga y Liu, 2017), lo cierto es que es una práctica cada vez más consolidada en todo tipo de retransmisiones televisivas, y que genera un enorme volumen de tuits. Los equipos de campaña son conscientes de que no deberán perder de vista este fenómeno, y no sólo durante el período electoral.

En los últimos años han ido apareciendo investigaciones sobre el comportamiento de Twitter durante los debates electorales televisados. Las primeras aparecen en Estados Unidos (Shamma, Kennedy, y Churchill, 2009) y Gran Bretaña (Chadwick, 2011; Vaccari, Chadwick, y O'Loughlin, 2015). A estas pronto se suman otras en Canadá (Elmer, 2012), Alemania (Trilling, 2015), Holanda (Vergeer y Franses, 2016), Italia (Bentivegna y Marchetti, 2015), Noruega (Kalsnes, Krumsvik, y Storsul, 2014), España (Ruiz del Olmo y Bustos Díaz, 2017) y surgen otro tipo de trabajos de carácter contrastivo, como el de Wells et al, (2016) en el que se comparan rasgos distintivos del discurso en la segunda pantalla en Francia y Estados Unidos.

En la línea ya apuntada por Nee (2013), algunas han confirmado que los usuarios más activos en el debate político en la segunda pantalla son también los que muestran un mayor compromiso cívico y político (Chadwick, O’Loughlin, y Vaccari, 2017; Vaccari et al., 2015; Vergeer y Franses, 2016). Chadwick, O'Loughlin, y Vaccari (2017) apuntan la existencia de diferencias de género, pues mientras la mujer dice emplear las redes para la búsqueda de información y la configuración de su voto personal, el hombre busca principalmente persuadir a otros, extender su influencia, hacer valer su criterio, al intervenir en la conversación de la segunda pantalla. Horsch-Dayican, Amrit, Aarts, y Dassen en su investigación sobre la campaña holandesa de 2012 señalan también este rasgo persuasivo de las intervenciones del ciudadano anónimo en Twitter, aunque probablemente lo más llamativo es el carácter negativo de gran parte de la conversación ciudadana en esta red: "It was interesting to see that negative messages have the biggest share among tweets from citizen users. The analysis of the contents of these tweets suggested that citizens' negative campaigning is rather an 
expressive act and in this way it differs from online persuasive campaigning efforts" (HoschDayican, Amrit, Aarts, y Dassen, 2014, p. 147).

El discurso que se crea en red (segundo nivel discursivo) sobre el debate electoral, encuentra en esos medios sociales, diferentes del medio de comunicación tradicional pero, como indica Campos Freire (2008) complementario de estos, un nuevo espacio de difusión diferente y diferenciado, pues se produce en otro entorno tecnológico que implica diferencias formales, contextuales, lingüísticas y de contenido.

Siendo conscientes de nuestras discrepancias con la restrictiva y ampliamente asentada definición de "discurso político" que Van Dijk formula como: "the discourse of politicians" (van Dijk, 2003, p. 212), y que se remonta a Aristóteles, el universo textual de tuits que versan sobre la política y que toman como referente conversacional un género político como el debate electoral podría ser también entendido como discurso político en un sentido laxo (Gallardo-Paúls, 2018). Beatriz Sarlo (2011) habla de la nitidez, brevedad y vivacidad de los enunciados como cualidades fundamentales de la expresión en redes, y de la ironía y el sarcasmo como elementos consustanciales. Gallardo-Paúls y Enguix-Oliver (2016) lo clasifican como "discurso pseudopolítico", argumentando que en las redes se produce un desplazamiento (Gallardo-Paúls, 2018) respecto a lo que el canon clásico ha defendido como discurso político. Este discurso sobre lo político se caracteriza por acentuar el personalismo, la desideologización y la espectacularización (Gallardo-Paúls y Enguix Oliver, 2016), aspecto sobre lo que otros coinciden (Vallespín, 2015). A nivel discursivo eso se traslada en una "producción logorreica, falta de estructura, condensación expresiva, sobreinterpretación, hipersignificación, hipermilitancia y encapsulamiento afiliativo"(Gallardo-Paúls, 2018, p. 10); hablamos así de una primacía de la función ilocutiva sobre la referencial o proposicional, la absorción del enunciado por la enunciación, una ilocutividad basada en la inferencia, la condensación expresiva o la ilocutividad expresiva sobre la representativa, y una mayor incidencia en los matices negativos del discurso. Este desentrañamiento de los rasgos lingüísticos que conforman el discurso pseudopolítico continúa en la línea del planteamiento de Mayer (2002), quien advertía de los riesgos que la mediatización de la política podría inferir al debate político. Es el riesgo del triunfo de la teatralidad, las apariencias y los gestos frente a la acción política real, a los hechos.

La siguiente tabla (tabla 1) explica qué rasgos lingüísticos se acentúan, y afianzan el personalismo, la desideologización y la espectacularización, encuadrando los diferentes momentos del proceso comunicativo.

Tabla 1. Rasgos lingüísticos que definen el discurso “pseudopolítico” según Gallardo-Paúls (2018).

\begin{tabular}{|c|c|c|c|}
\hline Estrategias de encuadre & Personalismo & Desideologización & Espectacularización \\
\hline LÉXICA & $\begin{array}{c}\text { Usos de primera } \\
\text { persona, léxico } \\
\text { valorativo,... }\end{array}$ & $\begin{array}{c}\text { Léxico valorativo más } \\
\text { que referencial, } \\
\text { coloquialismos }\end{array}$ & $\begin{array}{c}\text { Léxico hiperbólico; } \\
\text { connotación e inferencia; } \\
\text { indirección humorística }\end{array}$ \\
$\begin{array}{c}\text { DE SELECCIÓN } \\
\text { TEMÁTICA }\end{array}$ & $\begin{array}{c}\text { Los propios políticos (y } \\
\text { su "lado humano") } \\
\text { como tema }\end{array}$ & Temas no políticos & $\begin{array}{c}\text { Temas periféricos y } \\
\text { anecdóticos; trivialización } \\
\text { de los asuntos; predominio } \\
\text { de las soft news en los } \\
\text { medios }\end{array}$ \\
\hline
\end{tabular}


RLCS, Revista Latina de Comunicación Social, 76, 121-141

[Investigación] DOI: 10.4185/RLCS-2020-1440 | ISSN 1138-5820 | Año 2020

\begin{tabular}{|c|c|c|c|}
\hline PREDICATIVA & $\begin{array}{l}\text { El político como sujeto } \\
\text { sintáctico }\end{array}$ & $\begin{array}{l}\text { Personalización de los } \\
\text { temas, acción } \\
\text { dramática }\end{array}$ & $\begin{array}{l}\text { Dramatización, moralismo } \\
\text { y escándalo }\end{array}$ \\
\hline ESTRUCTURAL & $\begin{array}{l}\text { Filtro de los temas por } \\
\text { la narrativización } \\
\text { biográfica }\end{array}$ & $\begin{array}{l}\text { La política como } \\
\text { "relato" }\end{array}$ & $\begin{array}{l}\text { Discurso de acción más que } \\
\text { reflexión argumentativa }\end{array}$ \\
\hline INTENCIONAL & $\begin{array}{l}\text { Actos expresivos, } \\
\text { directivos y } \\
\text { compromisorios }\end{array}$ & $\begin{array}{l}\text { Actos expresivos } \\
\text { (sentimentalidad), } \\
\text { actos directivos, } \\
\text { performativos, } \\
\text { explícitos }\end{array}$ & $\begin{array}{l}\text { Actos negativos: expresivos } \\
\text { (quejas), directivos } \\
\text { (insultos, reproches), } \\
\text { compromisorios (amenazas, } \\
\text { intimidación) }\end{array}$ \\
\hline PARATEXTUAL & $\begin{array}{l}\text { Publicaciones con la } \\
\text { imagen de los políticos }\end{array}$ & Selfies, gifts y memes & $\begin{array}{l}\text { Selfies, gifts y memes. } \\
\text { Estrategias de ludificación }\end{array}$ \\
\hline INTERACTIVA & $\begin{array}{l}\text { Publicaciones de } \\
\text { autocita o de textos en } \\
\text { los que se habla del } \\
\text { propio político }\end{array}$ & $\begin{array}{l}\text { Predominio de la } \\
\text { discrepancia; } \\
\text { predominio de la } \\
\text { oposición vs. La } \\
\text { afiliación partidista }\end{array}$ & $\begin{array}{l}\text { Insultos y descortesía. En } \\
\text { los medios insistencia en lo } \\
\text { agonístico, en el } \\
\text { enfrentamiento entre } \\
\text { políticos }\end{array}$ \\
\hline
\end{tabular}

Fuente: extraído de Gallardo-Paúls (2018). Elaboración propia.

Tras revisar investigaciones previas sobre el discurso que genera el usuario de Twitter durante su visualización de producciones televisivas entre otras, algunos debates electorales en países de nuestro entorno, y analizar qué rasgos lingüísticos definen el discurso pseudopolítico, pasamos a presentar este estudio.

\section{Metodología}

El objeto de análisis de nuestra investigación es la conversación producida en Twitter en el primer debate electoral realizado en España con motivo de las elecciones generales del 28 de abril de 2019. Este, realizado el 22 de abril de 2019, se produce 26 años después del primer debate, aquel que tuvo lugar en el año 1993 entre los candidatos del PSOE y el PP, Felipe González y José María Aznar respectivamente, en las elecciones generales del 6 de junio de 1993. El que es objeto de este trabajo fue organizado por la televisión pública, Radio Televisión Española (RTVE) y transmitido a través de los canales: La 1, 24h, RNE, en sistema de streaming por RTVE.es, y por TVE internacional y Radio Exterior, y por otras televisiones como IB3, Castilla La Mancha Televisión, Canal Extremadura, Trece, Telemadrid, Canal Sur, Aragón TV y TPA 2. Su audiencia fue de 8.886.000 espectadores, lo que significó una elevada cuota de pantalla de un $43,8 \%$, por lo que fue lo más visto ese día (Barlovento Comunicación, 23/04/2019).

Teniendo en cuenta previas aproximaciones al tema, quisimos conocer el interés que pudieron tener los partidos en intervenir y orientar la conversación sobre el debate electoral en el espacio de esa segunda pantalla, y la orientación comunicativo-lingüística que ese debate tuvo entre los prosumers que marcaron tendencia en esa conversación. Por ello planteamos las siguientes preguntas de investigación: 
1. ¿Intervinieron todos los partidos presentes en el debate electoral del 22 de abril de 2019 en la conversación en Twitter?

2. ¿La mayoría de las intervenciones de estos partidos fueron de ataque al adversario?

3. ¿El discurso político creado en la conversación de esta red social durante el debate podría calificarse propiamente como político o más bien como pseudopolítico?

4. ¿Las fórmulas expresivas de ese debate paralelo tendieron en gran medida hacia la mofa y broma?

Este trabajo se enmarca en la tradición de estudios pragmático-discursivos y encuentra sus referentes dentro de la lingüística cognitiva. El análisis del discurso orienta nuestra investigación. En esta, empleamos el modelo de análisis de base pragmática de Gallardo-Paúls $(2013,2014)$ que esta autora ha aplicado al análisis de varios tipos de corpus. Este bebe de autores clásicos como Austin (1962), Searle (1969) o Wodak (2001) entre otros, se basa en los presupuestos de la lingüística cognitiva de Lakoff $(1990 ; 1980)$ y Fillmore $(1985 ; 1976)$ e incide en la relevancia del encuadre en el momento de la emisión, en el texto en sí mismo o enunciado y en el destinatario. Aquí hemos analizado el primer momento, el de la enunciación. Hemos de apuntar también que estamos de acuerdo con Piñuel y Gaitán (1995, p. 516) cuando señalan que: "podría plantearse si lo que se entiende por Análisis Textual o Análisis del Discurso no es sino una modalidad del Análisis de Contenido, o viceversa".

Como bien explica Ibarretxe Antuñano (2013), la lingüística cognitiva entiende el lenguaje como parte de las capacidades cognitivas del ser humano, entre las que también se encuentran la memoria, el razonamiento, la categorización y la atención y actúa combinada con ellas. Es un proceso que afecta a los dos planos del habla señalados por Benveniste (1958): el del enunciado y la enunciación, $\mathrm{y}$ desde una perspectiva pragmática a sus tres niveles: el enunciativo, el textual y el interactivo (Gallardo-Paúls, 1996).

Como ya hemos apuntado, en esta investigación quisimos conocer los aspectos cognitivos que actúan en el emisor y le hacen encuadrar sus mensajes en Twitter, por lo que atendimos al nivel enunciativo exclusivamente, dejando de lado los niveles textuales o interactivos.

La ficha de análisis se compone de las categorías que se ven en la tabla 2. Analizamos en primer lugar la estrategia léxica a través del análisis de los hashtags empleados en el tuit (si eran connotativos o denotativos) y respondiendo a si necesitábamos hacer uso de la inferencia o no para comprender el tuit. En segundo lugar, atendimos a la estrategia temática de los tuits. Por un lado, nos preguntamos si la temática era política o no política y, cuando sí era política, de qué hablaban esos tuits.

Para ello distribuimos los temas según varias categorías. Unas estaban relacionadas con la Policy issues según la define Patterson (1980, en Mazzoleni, 2014, p. 210), es decir analizamos si los tuits trataban de: (1) Política económica, fiscal y empleo; (2) Política social, Estado del bienestar, pensiones e igualdad; (3) Política territorial y (4) Regeneración democrática y pactos postelectorales. Este último punto, pactos post-electorales formaría parte, según Paterson, de las Political issues, aunque nosotros la analizamos conjuntamente con el punto de "Regeneración democrática" para seguir las líneas temáticas establecidas en el debate de RTVE. A estas cuatro categorías añadimos otra denominada (5) "Opiniones sobre las elecciones, los candidatos, etc.", a la que vinculamos los tuits relacionados con las elecciones, los sondeos, los candidatos, etc., es decir aquello denominado por Patterson como Campaign issues. En la categoría de "Temas no políticos" a la que hemos hecho referencia antes, se incluyen asuntos que, siguiendo a Patterson (1980, citado por Mazzoleni, 2010) tienen más que ver con "Personal Issues", es decir con la vida y actividad de los 
candidatos. Aquí también se incorporaron las burlas, comentarios irónicos o figuras retóricas difícilmente clasificables en ningún otro apartado.

En la estrategia intencional tratamos de ver cuál era la ilocutividad de los actos de habla producidos en el tuit. Para ello empleamos la clasificación clásica de Searle (1969), separando además los actos de habla expresivos en dos tipos: positivos o negativos. Dentro de esta estrategia, también analizamos la orientación observada en el tuit, que se desagregó en tipos de ataque o tipos de elogio (siguiendo la propuesta empleada por Benoit (1999) para otro tipo de corpus de carácter eminentemente político), diferenciados según la agrupación política a la que iban dirigidos. Finalmente, la estrategia predicativa respondía a la pregunta de quién fue el enunciador del mensaje. En este punto se clasificaron las cuentas según dos criterios: (1) aquellas que respondían a un apodo ("con apodo") o que incluían una selección aleatoria de letras, y (2) aquellas otras que calificamos como "identificadas" y donde se incluyeron las pertenecientes a los partidos, sus candidatos principales o sus agrupaciones locales, autonómicas, etc., las pertenecientes a medios de comunicación o periodistas, y las de usuarios identificados con nombre y apellidos. (Ver Anexo 1).

Tabla 2. Modelo de análisis con categorías hasta el primer nivel.

\begin{tabular}{|c|c|c|}
\hline \multirow{7}{*}{$\begin{array}{c}\text { Encuadre } \\
\text { enunciativo }\end{array}$} & \multirow{2}{*}{ Estrategia léxica } & Etiquetas \\
\hline & & Inferencias \\
\hline & \multirow{2}{*}{$\begin{array}{l}\text { Estrategia } \\
\text { temática }\end{array}$} & Temática \\
\hline & & Partido objeto del tema \\
\hline & \multirow{2}{*}{ Estrategia intencional } & Ilocutividad \\
\hline & & Orientación \\
\hline & Estrategia predicativa & Agentividad \\
\hline
\end{tabular}

Fuente: elaboración propia.

El corpus lo componen 1000 tuits seleccionados de forma aleatoria por el programa de análisis cualitativo Atlas.ti de entre los más populares del debate del 22 de abril. La selección se hizo tomando como referencia los hashtags publicitados por Televisión Española para la generación de la conversación en red, es decir: \#ElDebateEnRTVE y \#ELDEBATEeenRTVE. Al contrario que en otros casos (Anstead y O'Loughlin, 2011; Kalsnes et al., 2014; Shamma et al., 2009) y siguiendo el ejemplo de investigaciones como las de Trilling (2015) limitamos la investigación a las etiquetas propuestas por la cadena, evitando así los "falsos positivos" que podrían haber lastrado el estudio y entorpecido nuestro trabajo.

La versión 8 de Atlas.ti permite importar un máximo de 1000 tuits, que se pueden seleccionar según varios criterios; en nuestro caso establecimos en cuanto al tipo de resultado los tuits más populares y, sobre los datos, en el corpus definitivo se incluyen retuits e imágenes. 
En 2011 Anstead y O'Loughlin se preguntaban por la significatividad de una muestra reducida de tuits cuando el universo muestral susceptible de análisis es tan extenso. Coincidimos con ellos y con Chadwick (2011) cuando apuntan que: "we also need to be mindful of the possible number of people reading Twitter content, which would multiply the importance of the content by a great magnitude" (Anstead y O’Loughlin, 2011, p. 447). Además, reivindicamos la influencia que Twitter tiene a día de hoy sobre los periodistas, los medios y la política como fuente documental y de noticias, lo que lo convierte en un instrumento de referencia. Y precisamente son los tuits más populares los que generan opinión pública. Entendemos que el criterio de popularidad es lo suficientemente significativo para que este sea un corpus válido y fiable.

El universo de análisis del discurso paralelo difundido en Twitter con motivo de este debate fue de $\mathrm{n}=107.915$ tuits, por lo que nuestra muestra supone tan solo un $0,93 \%$. Para conocer el universo de análisis, empleamos un script programado en Python "Get Old Tweets Programatically" (JeffersonHenrique, 2016).

La codificación de los tuits la llevó a cabo un investigador en Atlas.ti y se emplearon tablas de coocurrencias para extraer los resultados, que se procesaron y analizaron también en Microsoft Excel.

\section{Resultados}

Uno de los resultados más visibles del análisis es que hasta un 70,6\% $(\mathrm{n}=706)$ de la muestra fueron retuits, es decir que no eran contribuciones escritas ex profeso por el prosumidor (Toffler, 1980), reflexiones propias, aforismos o pensamientos originales, sino que eran mensajes de autoría ajena y redifundidos por el usuario desde su cuenta, lo que muestra la alta "viralidad" de estos contenidos, en el sentido ya apuntado por Roncallo-Dow, Córdoba-Hernández y Durán Camero (2019, p. 129) o del-Fresno-García y Daly (2019, p. 70). A su vez, ello apunta a la escasa proactividad creativa de estos usuarios.

La primera pregunta que tratamos de responder es si todos los partidos presentes en el debate televisado intervinieron en esa otra conversación en Twitter, lo que el análisis confirmó. En una primera aproximación, Atlas.ti apuntó la existencia de hasta 44 intervenciones retuiteadas de la cuenta de@populares, 39 del@PSOE,30 de@ahorapodemos y 29 de la cuenta @CiudadanosCs, mostrando mayor actividad así el primer partido mencionado. Sin embargo, sus intervenciones reales, es decir, no solo las procedentes de las cuentas oficiales fueron más elevadas. Para conocer el alcance de la intervención de los partidos en el debate en la segunda pantalla vinculamos a otros usuarios próximos a las organizaciones políticas (candidatos, organizaciones autonómicas o locales, etc.) con su organización; por ejemplo las intervenciones de @ cayetanaAT (cuenta de Cayetana Álvarez de Toledo) fueron incorporadas al PP. Para conocer la estructura predicativa, fijamos nuestra atención en la procedencia autoral del tuit (en los casos de retuit ello se analizó como autoría del "segundo enunciatario"). Así, encontramos que los partidos intervinieron en el debate de la segunda pantalla, pero en unos porcentajes relativamente bajos respecto a la muestra total, pues ninguno de ellos superó el $10 \%$.

El partido más presente en este debate en redes fue Unidas Podemos, que a través de su propia cuenta y otras asociadas intervino con un $8,9 \%(n=63)$; pero todos participaron en mayor o menor medida, por ejemplo, las cuentas vinculadas al PP, con un 5,4\% $(n=38)$; al PSOE, con un 4,8\%, $(n=34)$ y a Cs con un $4,5 \%,(n=32)$.

Todas estas cuentas vinculadas a partidos fueron analizadas bajo la categoría de "Cuentas identificadas", donde según hemos ya visto también se incluyeron las cuentas de usuarios anónimos 
pero que en Twitter se identificaban con nombre y apellido, y cuentas de periodistas o medios de comunicación $(\mathrm{n}=80 ; 11,4 \%)$. En conjunto, la mayor parte de las intervenciones partieron de estas "Cuentas identificadas" $(70,4 \%, n=704)$, de las cuales la mayor parte procedía de usuarios no adscritos a partido o medio de comunicación alguno. Ver gráfico 1.

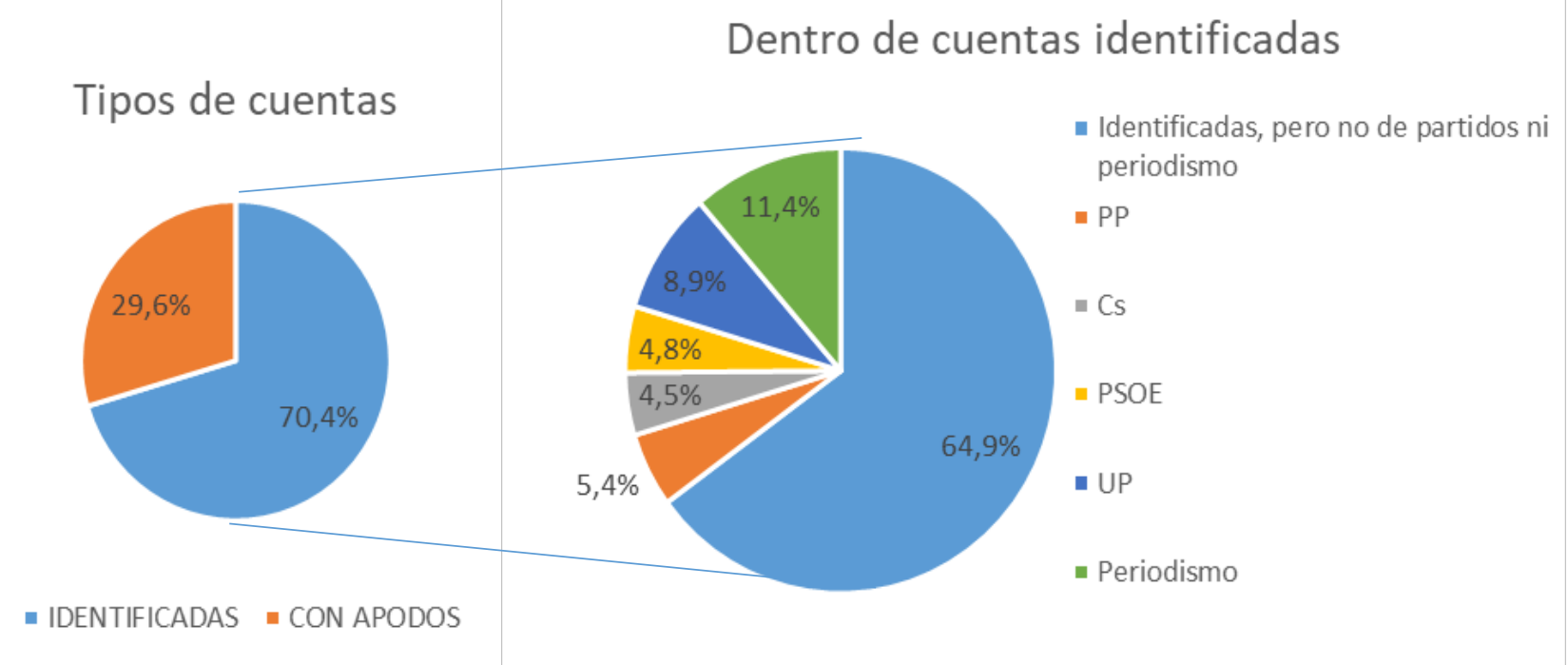

Gráfico 1. Tuits según el tipo de cuentas analizadas.

Fuente: elaboración propia.

En segundo lugar quisimos saber si las intervenciones de los partidos en Twitter fueron principalmente de ataque al adversario es decir, si en su intento de dirigir la conversación en Twitter sobre el debate, el ataque al adversario había sido su principal objetivo. Sabemos que estudios sobre la comunicación política como los de Benoit (1999, p. 20) han analizado el discurso político según este proponga aclamaciones, ataques o defensas al candidato o partido. Teniendo en consideración aquel modelo, y dentro de la estrategia intencional, tratamos de conocer cuál había sido la orientación del tuit. Como hemos visto previamente las opciones eran tres: el ataque al adversario (especificando de qué adversario hablaba), el elogio o aclamación (con similar distinción) y la no existencia de orientación clara. Los resultados de ese debate en red muestran que el ataque al adversario no fue la opción preferida por las cuentas vinculadas a los partidos en Twitter excepto en un caso: el de Ciudadanos.

Las cuentas vinculadas a PP, PSOE y UP difundieron mensajes sobre todo de elogio, aclamación y apoyo a su candidato, con diferente gradación, pues en las cuentas vinculadas a PP ese apoyo fue muy significativo, de hasta un $85 \%(n=34)$, algo menor en las vinculadas a PSOE $(70,2 \%, n=33)$ y no superó el $50 \%$ en el caso de las vinculadas a Unidas Podemos $(41,9 \%, \mathrm{n}=31)$.

La estrategia en Twitter de las cuentas vinculadas a Cs fue principalmente de ataque al PSOE $(54,8 \%, n=17)$ antes que el elogio propio $(35,5 \%, n=11)$ aunque, al observar los porcentajes, se ve mayor variación y que los resultados no son tan rotundos.

Aunque el ataque no fue la estrategia más empleada por los partidos, sí caracterizó la conversación general en esa segunda pantalla de \#EIDebateenRTVE, pues más de la mitad de las intervenciones $(52,1 \%, \mathrm{n}=578)$ de los prosumers fueron de ataque, ya fuera a partidos, periodistas o en general, como se puede ver en la gráfico 2 . 


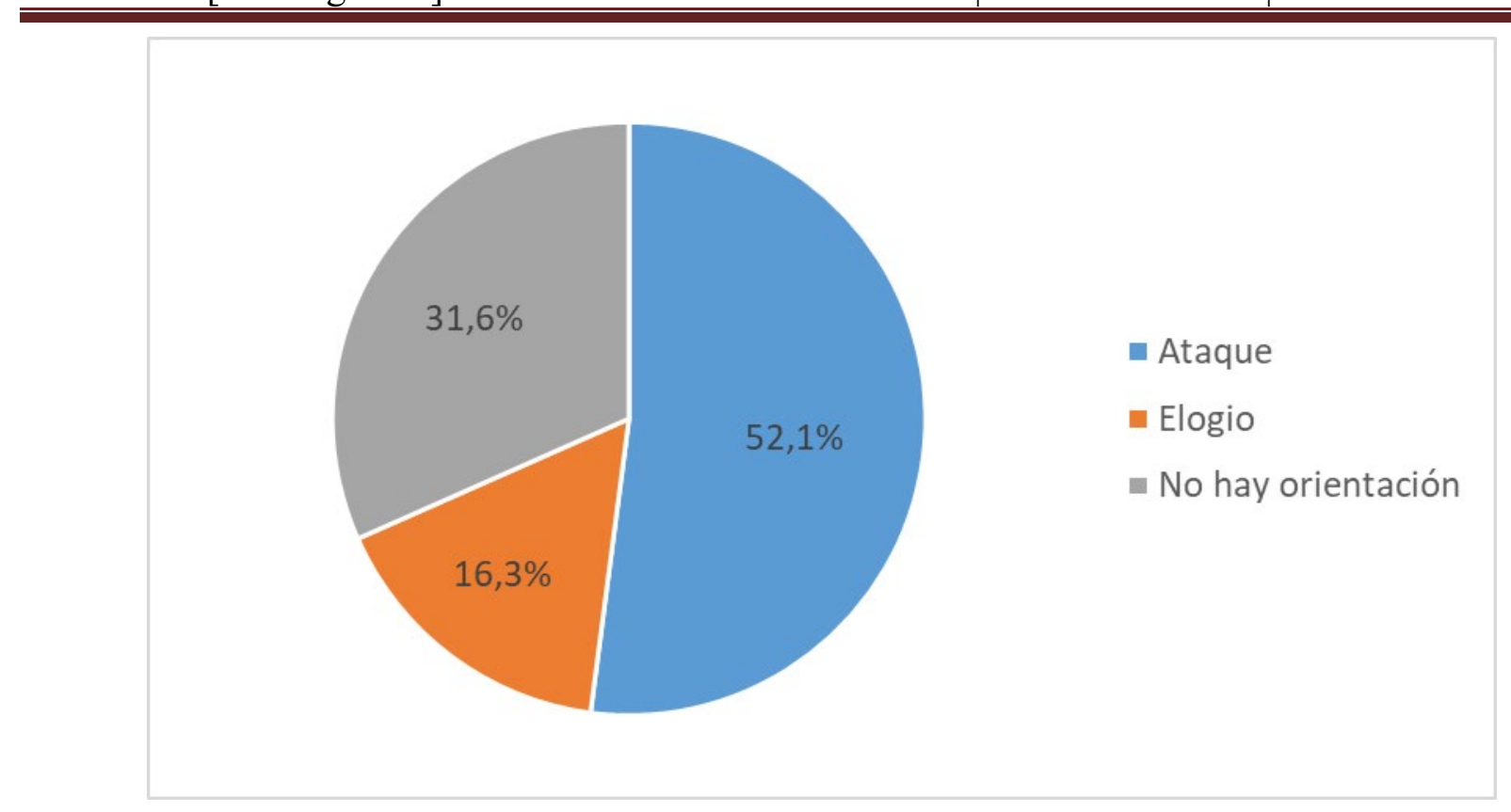

Gráfico 2. Estrategia intencional del tuit según su Orientación.

Fuente: elaboración propia.

El análisis de la ilocutividad apunta matices diferentes según hablemos de cuentas vinculadas al Partido Popular, en el que priman las intervenciones de carácter "compromisorio" $(47 \%$; $n=18)$, de ilocutividad representativa en el caso de Unidas Podemos $(51 \%$; $n=32) \mathrm{y}$, en los casos de Ciudadanos y PSOE, la ilocutividad preferente es la "Expresiva negativa" (Cs: 63\%, n=20; PSOE: 47\%, n=16). En el caso del PP esa ilocutividad compromisoria se ejemplifica en este tuit (Imagen 1), en el que se recurre a hipérboles como la de "la mayor revolución fiscal de la historia", transmitida a través de la primera persona del singular.

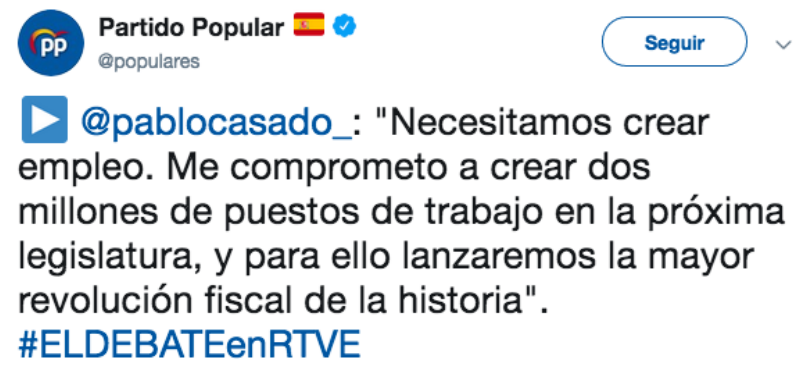

\section{Imagen 1.}

Fuente: captura de pantalla de Twitter realizada el 1 de octubre de 2019.

El candidato de Cs también prefirió emplear esa persona verbal, accionando así estrategias para la personificación de su discurso.

La ilocutividad representativa de las cuentas de Unidas Podemos se explica por las numerosas ocasiones en las que emplean el discurso referido en esta otra conversación en red, como en el ejemplo que se ve a continuación (imagen 2). 


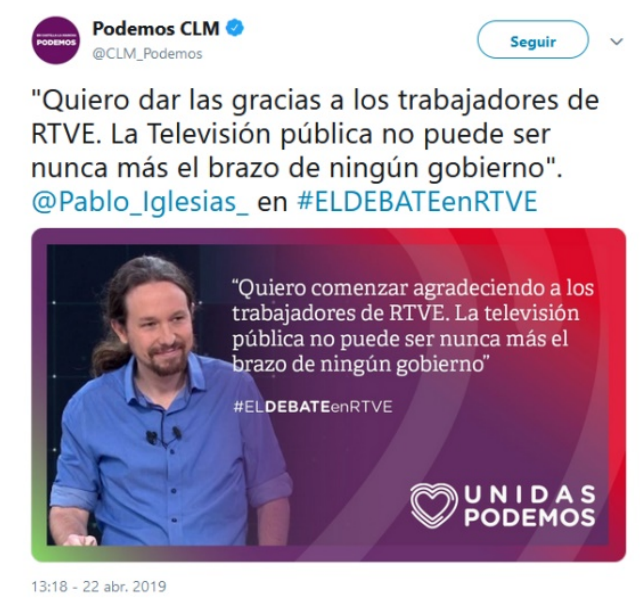

Imagen 2.

Fuente: captura de pantalla de Twitter realizada el 1 de octubre de 2019.

Volviendo al corpus general, los tuits de expresividad negativa son los que imperan en la segunda pantalla, si bien a corta distancia de la ilocutividad representativa fruto, como acabamos de ver, de la cita directa del discurso del emisor en televisión.

Para responder a la tercera pregunta, es decir, para conocer si el tipo de discurso sobre el debate en Twitter estaba alejado de los cánones que han definido el discurso político como tal (van Dijk, 2003; Charaudeau, 2005), fijamos nuestra atención en variables como: el uso del hashtag y la inferencia (que construyen la estrategia léxica), la agenda temática de los tuits y el protagonismo del partido (correspondientes a la estrategia temática) y la ilocutividad y la orientación de esos escasos caracteres, muchas veces completados con enlaces, imágenes y/o vídeos (que daban forma a la estrategia intencional).

El uso de los hashtags fue escaso, lo que confirma algo apuntado por estudios previos (Boyd, Golder, y Lotan, 2010); tan solo se emplearon en un $24,4 \%$ de las ocasiones $(n=244)$. Cuando estas etiquetas aparecen son fundamentalmente de tipo connotativo $(18 \%, n=180)$, lo que indica una intencionalidad persuasiva. Gran parte de estas etiquetas connotativas respondían a los hashtags de campaña o los elegidos por los partidos para jalear a sus candidatos en el debate, es decir los: \#GanaPedro, \#VamosAlbert, \#ValorSeguro, \#LaHistoriaLaEscribesTú. Estas compartían espacio con otras como \#DebateCTXT con las que algunas empresas periodistas o medios quisieron dar continuidad a una conversación textual convirtiéndola en transmediática. Por supuesto el uso de los hashtags no solo nos ofrece un marco de interpretación del mensaje sino que su uso conlleva la participación en una comunidad con intereses similares (Bentivegna y Marchetti, 2015; Zappavigna, 2011).

Las inferencias estuvieron muy presentes en el corpus, pues más de la mitad de los tuits exigían tener un conocimiento previo del contexto político nacional para dar un sentido lógico al texto. A veces la ayuda gráfica en forma de video o imagen favorece la interpretación del tuit, pero otras veces la hace más compleja y llena de matices.

Esos tuits cargados de inferencias y presuposiciones ocupan un 55,2\% de la muestra, porcentaje algo menor que el de otras investigaciones (Vergeer y Franses, 2016) pero significativo, que nos hace pensar que este debate de la doble pantalla dialoga sobre el debate original, creando un entramado 
textual que solo los elementos formales (hashtag, hilos, etc.) y el propio contexto permiten desentrañar.

Para conocer si la agenda temática presente en \#EIDebateEnRTVE tenía relación con la del debate televisado o si ese otro debate adoptaba otra agenda diferenciada analizamos los tuits del debate en la segunda pantalla según su relación con alguna de las cuatro temáticas en las que se dividió el debate en la cadena pública, de las que hemos ya hablado.

La conversación en red resultó estar más centrada en cuestiones secundarias al hecho político y las políticas de interés ciudadano que en aquellos temas tratados en el debate televisado; de hecho, el mayor número de tuits trató sobre "Opiniones sobre las elecciones, los candidatos, etc.", $(36,5 \%)$ y temas estrictamente no políticos $(25,2 \%)$. (Ver gráfico 3$)$

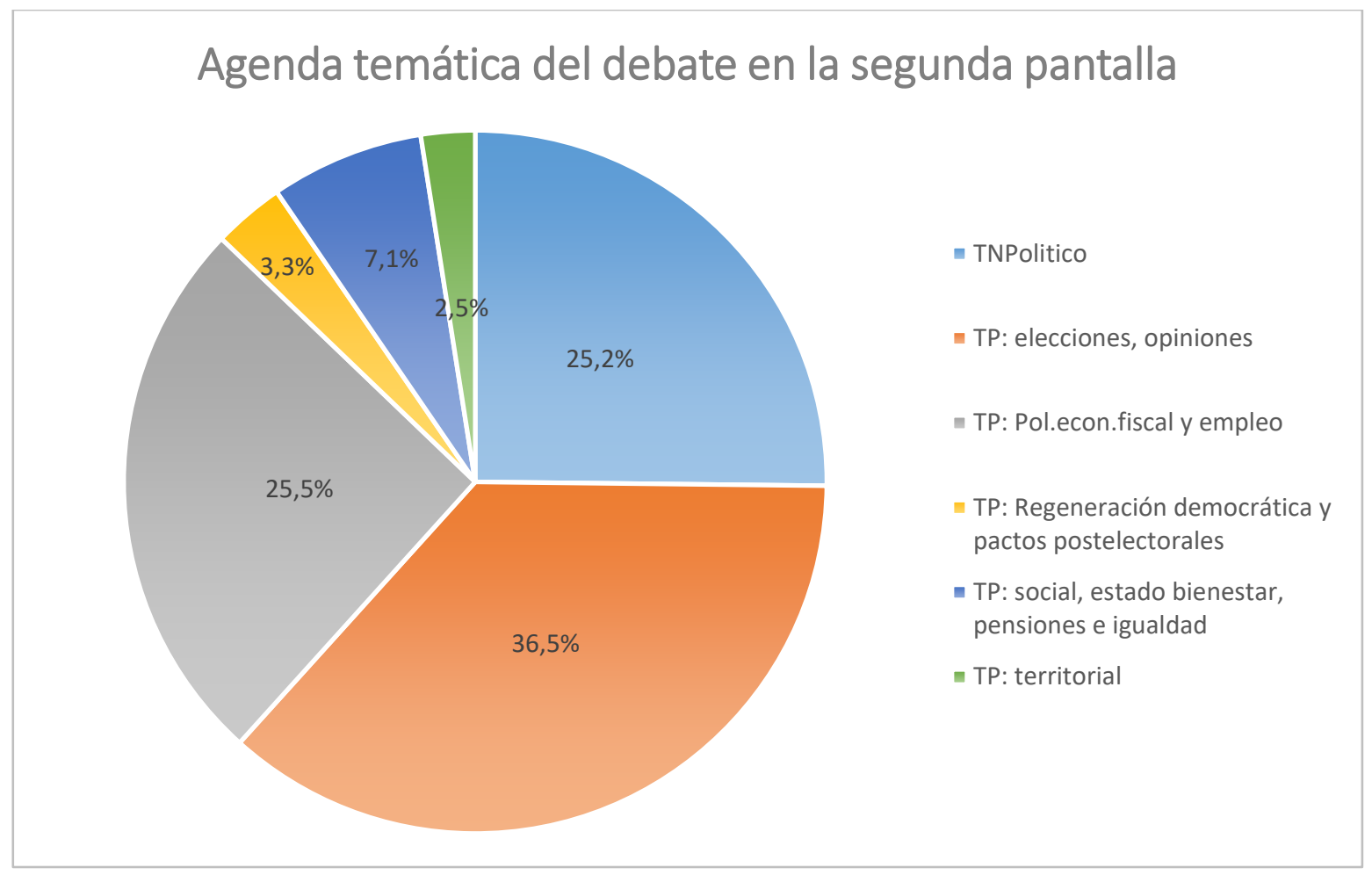

Gráfico 3. Agenda temática del debate en Twitter en porcentaje.

Fuente: elaboración propia.

De los cuatro grandes bloques temáticos del debate, el que mayor número de intervenciones generó en Twitter fue el de Política económica, fiscal y empleo (25,5\%), a gran distancia del segundo tema, Política social, Estado del bienestar, pensiones e igualdad: 7,1\%, de la Regeneración política y pactos post-electorales: 3,3\%; o las Políticas territoriales $(2,5 \%)$; estas tres últimas con resultados casi anecdóticos. Observamos así como el contenido del debate en Twitter no giró precisamente alrededor de los temas tratados en el debate original pues, si bien el nuestro no es un estudio contrastivo, el hecho de que el porcentaje de tuits relacionados con la agenda temática propia de lo tratado en el debate televisado sea tan reducida, obliga a plantearnos si realmente Twitter sirvió o no como instrumento de deliberación y discusión constructiva, a lo que apuntaba Trilling (2015). De hecho, este autor señala la dificultad de que un tema tratado por un candidato en el debate televisado sea llevado a la conversación en la red en la forma positiva o negativa como el candidato desea sea este considerado, subrayando así la distancia entre ambas conversaciones y la práctica imposibilidad de que algún candidato pueda influir en la conversación de esta red social (Trilling, 2015, p. 272). 
Si tratamos de ver la agenda temática planteada por las cuentas vinculadas a cada uno de los partidos, los resultados son diferentes. Mientras las cuentas relacionadas con el partido de Pablo Casado tuitearon principalmente sobre Política económica, fiscal y empleo (95\%), las vinculadas a Ciudadanos se repartieron y, si bien dedicaron a ese tema bastante atención (un 41\%), también escribieron sobre Regeneración democrática y pactos post-electorales en gran medida (34\%) y opinaron sobre elecciones y candidatos $(22 \%)$. Las cuentas vinculadas al partido de Pablo Iglesias dedicaron mucho espacio a ese tema de la "Política económica, fiscal y empleo" (70\%), y algo menos al tema secundario de "Opiniones sobre las elecciones, los candidatos, etc." (13\%) y al de Política social, Estado del bienestar, pensiones e igualdad" (11\%). Y precisamente este último fue el más tratado por las cuentas vinculadas al PSOE (44\%), por encima de su interés por las "Opiniones sobre las elecciones, los candidatos, etc.” (31\%) y la Política económica, fiscal y empleo que tantas intervenciones provocó en las cuentas de PP y Unidas Podemos.

Una parte de las noticias que al día siguiente resumían el debate electoral del 22 de abril en los periódicos, radios y televisiones lo presentaban a través de una imagen: los memes que habían surgido en esa conversación (ABC, 23/04/2019; FCINCO, 23/04/2019; G.C., 22/04/2019; El Heraldo, 23/04/2019; Justo, 23/04/2019; LOM, 23/04/2019; Redacción Yo Tele, 23/04/2018; Verne, 23/04/2019). La anécdota contenida en los memes, en los tuits con contenido irónico, o burlesco ocupaba páginas en prácticamente todos los medios del país. Seguramente porque, como indica Cortázar Rodríguez, a día de hoy: "Los memes de internet son considerados fragmentos de cultura que contienen ideas y que van influenciando a la gente en la medida en que se propagan (exagerando en ocasiones la influencia inmediata que podrían tener sobre las actitudes y los comportamientos de la gente)" (2014, p. 204).

Visto esto, la última de las hipótesis del trabajo fue constatar que ciertamente la red social creada por Jeff Dorsey dedicó gran parte de sus textos a este tipo de textos irónicos o burlescos, como habían apuntado algunos estudios previos (Anstead y O'Loughlin, 2011; Freelon y Karpf, 2015; Trilling, 2015). De hecho, en alguna de estas investigaciones la prevalencia era muy elevada: "In the case of Twitter reactions to the first and third 2012 Presidential debates, political humour was quite prevalent. Fifty-eight per cent of the most-duplicated tweets across both debates contained humour" (Freelon y Karpf, 2015, p. 402).

Sin embargo, nuestro análisis indica que este tipo de intervenciones en las que se emplean la mofa, la broma y el humor dando forma a los memes no fueron excesivamente altas. Estas fórmulas textuales humorísticas tan solo constituyen el 16,1\% $(\mathrm{n}=161)$ de la muestra, y ello considerando un aspecto esencial en su construcción, y es que esta rescató las 1000 intervenciones más populares, esas donde a juicio de Freelon y Karpf (2015) más suelen aparecer. Tras estos resultados dirigimos nuestra mirada a los medios de comunicación tradicionales, que tanto se interesaron y hablaron de los memes creados ex profeso durante el análisis del debate en Twitter y nos preguntamos por los criterios que emplean en la generación de su agenda, por sus criterios de noticiabilidad y su papel como gatekeepers de la información.

\section{Discusión y conclusiones}

Dos fueron los debates electorales que los candidatos de los principales partidos políticos con presencia en las Cortes Generales de la XII Legislatura protagonizaron: el emitido por RTVE que tuvo lugar el 22 de abril de 2019 y el de Atresmedia un día después. La conversación mantenida en la red social Twitter por partidos y usuarios y recogida bajo las etiquetas oficiales fue el objeto de nuestra investigación. El corpus analizado forma parte así de un "ambiente de filiación", y de una "conversación que puede buscarse" en el sentido señalado por Zappavigna (2011), pues gracias al 
uso de las etiquetas una audiencia geográficamente dispersa participó en una conversación que trascendió lo inmediato, y permitió a las audiencias del debate -y a otros que no lo siguieron- seguir una conversación en la que la pantalla televisiva era el referente.

El debate en la segunda pantalla que hemos analizado contó con un elevado número de retuits, en la línea apuntada por otros estudios (Bentivegna y Marchetti, 2015; Guerrero-Solé y Mas-Manchón, 2017). Sin embargo, otros trabajos apuntan resultados diferentes; es lo que ocurre con el estudio de Vergeer y Franses (2016), lo que quizá podemos interpretar como un sesgo de nuestra investigación que, recordemos, trabajó sobre los tuits más populares, o puede tener relación con las diferencias culturales existentes entre Holanda y España. De cualquier forma, nuestros datos confirman la gran presencia de la práctica del retuiteo también en el comentario y visualización de un programa eminentemente electoral y político como es el debate. A través de esta práctica el usuario se expresa, participa, y construye su identidad virtual, pero no de forma tan activa, creativa y reflexiva como lo haría generando productos textuales propios.

Como supusimos, los partidos políticos presentes en el estudio de RTVE quisieron también estar presentes y transmitir su mensaje en esa otra conversación virtual paralela en Twitter y participaron. Lo hicieron a través de cuentas de destacados miembros, la del propio partido y sus organizaciones autonómicas y/o locales. Su participación en este discurso de la segunda pantalla les permitió trasladar su mensaje en esta red social, en la que pusieron en marcha estrategias clásicas de la comunicación política como el ataque al adversario o la aclamación del propio candidato, para tratar de conseguir que su mensaje llegara a todos los colectivos sociales, también a aquellos que no estaban viendo el debate electoral en pantalla, pero sí lo siguieron a través de las redes. De esta forma consiguieron la desintermediación en el flujo informativo (Bentivegna y Marchetti, 2015), y se erigieron en voces autorizadas mientras se confunden con el colectivo. Sin embargo, su participación en esta conversación no superó el $10 \%$. Sería interesante conocer si esta presencia mantiene estas características en futuros debates electorales.

Sin embargo las intervenciones procedentes de cuentas de usuarios vinculados a PP, PSOE, UP y Cs fueron mucho menores que las de ciudadanos sin vinculación política y/o periodística conocida (64,9\%), lo que coincide con Hosch-Dayican et al. (2014) y López-Meri (2017).

La estrategia intencional planteada por los partidos políticos en su encuadre discursivo en Twitter fue bastante similar en los casos de PP, PSOE y UP pero diferente en el caso de Ciudadanos. Como ya apuntamos, aquellos primeros optaron por elogiar a su candidato a través de sus cuentas vinculadas con diferente gradación, pues las cuentas vinculadas a PP y a PSOE hicieron un amplio trabajo de personalización y reivindicación del liderazgo de Pablo Casado y Pedro Sánchez, respectivamente. Por otra parte, la acción prioritaria de las cuentas de Ciudadanos fue el ataque al adversario, sobre todo el ataque al partido en el gobierno y su candidato, Pedro Sánchez. Sería interesante en este punto saber si la estrategia fue organizada por los equipos de campaña o producto de la iniciativa personal de miembros y colectivos políticos, pero ello exige otro tipo de aproximación metodológica que no era objeto de este estudio. Sin embargo y si bien aquella fue la estrategia de los partidos, el ataque fue la opción textual prioritaria del debate en red en su conjunto, lo que coincide con el estudio de Hosch-Dayican et al. (2014) sobre el comportamiento ciudadano en Twitter durante la campaña holandesa a las elecciones de 2012.

El debate de la segunda pantalla mostró que más allá del hashtag que sirvió para vehicular la conversación (\#ElDebateEnRTVE) el uso de etiquetas no fue muy elevado (Villar-Hernández, 2019) aunque cuando estas aparecen lo hacen mayoritariamente para activar un marco evaluativo, y con un objetivo persuasivo claro: evaluar positiva o negativamente a alguno de los candidatos. Los temas de 
este debate tuvieron una distribución temática que sabemos que no guardó paralelismo con el debate del estudio de televisión, aun sin haber nosotros analizado el debate en televisión. El motivo es claro, y es que tan solo uno de los temas tratados por los políticos, la Política económica, obtuvo una relevancia significativa en el debate en Twitter. Como vimos, la mayor parte de la conversación de la segunda pantalla versó sobre temas opinativos (sobre elecciones y candidatos) y temas no políticos, por lo que las políticas programáticas de los partidos, los compromisos que en el otro espacio se estaban enunciando, no tuvieron apenas peso en Twitter. Coincidimos con Trilling en que parte del contenido de Twitter estuvo influido por temas que en el debate televisado pasaron desapercibidos (Trilling, 2015). Por otro lado, ese tratamiento temático del debate no se distancia tanto del de los medios de comunicación clásicos, según García Marín (2015), quien apuntaba al soporte tecnológico como variable explicativa de ese sesgado comportamiento, lo que es un condicionante evidente del discurso en una red social como Twitter.

La elevada necesidad de contextualizar los 280 caracteres del mensaje en Twitter para conseguir una comprensión ajustada del texto en su conjunto fue otra de las características de la conversación, lo que nos hace reivindicar la etiqueta de "discurso pseudopolítico" en la definición de esta situación comunicativa concreta.

El hecho de que las etiquetas fueran mayoritariamente de tipo connotativo, unido a la elevada necesidad de contextualizar los tuits para seguir el discurso y el que la conversación tratara más de temas secundarios que de los propios del debate, nos permite afirmar que el debate en la red fue de tipo pseudopolítico, si bien esta realidad no es tan evidente como su análisis en otros tipos de corpus plantea (Gallardo-Paúls, 2017). Sin embargo, hay rasgos que apuntan a la existencia de personalización (alta incidencia de actos expresivos), desideologización (presencia de temas no político abundante, y actos de habla expresivos) y espectacularización (alta prevalencia de la inferencia y la presencia de la ironía, burla y sarcasmo) en el conjunto general del corpus analizado.

Evidentemente esa necesidad de aplicar constantemente la inferencia para la decodificación textual lleva a pensar en la facilidad de que un mensaje sea interpretado de maneras diferentes, según los puntos nodales propios del lector. Eso facilita a su vez la confusión interpretativa y la desideologización, pues vacía de significado absoluto el texto.

La presencia de los memes, el humor y lo humorístico tan prevalente en otras latitudes (Anstead y O'Loughlin, 2011; Freelon y Karpf, 2015) no fue especialmente significativa en nuestro corpus, pese a que la mayor parte de los medios de comunicación tradicionales se hicieran eco al día siguiente de su existencia.

La presente investigación inicia una línea de trabajo poco desarrollada en el contexto español: el estudio empírico de la interacción en Twitter sobre el debate electoral en el contexto de unas elecciones generales a la Presidencia del Gobierno español, en particular el producido el 22 de abril de 2019. Se ha descrito que los cuatro partidos presentes en el debate televisivo participaron, si bien no de forma muy relevante, en ese otro que se produjo en la segunda pantalla, y que sus intervenciones se dirigieron sobre todo a elogiar la actuación y las políticas de sus propios candidatos con una excepción, Ciudadanos, partido que optó por el ataque al PSOE y su candidato. Con este estudio se inicia una línea de trabajo que pretende conocer las estrategias comunicativas de los diferentes partidos entorno al debate paralelo creado en la red social Twitter.

En cuanto al conjunto de prosumers que participaron en este otro debate, sus intervenciones sí fueron mayoritariamente de ataque, tal y como estudios en países de nuestro entorno han apuntado (e.g. Holanda). Por otro lado, gran parte de ese discurso podría ser calificado como pseudopolítico. Sin 
embargo los memes, el humor y lo humorístico no constituyeron una parte esencial y central del mismo. Una continuidad longitudinal de este estudio sería interesante para ver si los resultados obtenidos en este trabajo se mantienen o se producen variaciones, pero con el necesario estudio de técnicas de investigación alternativas que permitan una codificación más ágil de una muestra más elevada de la población de tuits.

La inestable realidad política española, a la que a día de hoy debemos sumar unas nuevas elecciones en 2019 (el 10 de noviembre) ha hecho que en estos momentos podamos sumar nuevos debates electorales entre candidatos a la Presidencia del Gobierno, consolidando así este fenómeno televisivo y su correlato textual en redes sociales. Twitter se ha convertido en una herramienta de visibilidad política, otra plataforma de distribución más de contenidos con rasgos propios y diferenciados donde se juega un partido diferente, pero partido que ganar o jugar al fin y al cabo.

\section{Referencias bibliográficas}

ABC (23/04/2019). Los «memes» que ha dejado el primer debate electoral. https://www.abc.es/recreo/abci-memes-dejado-primer-debate-electoral201904231010 noticia.html

Anstead, N. \& O'Loughlin, B. (2011). The Emerging Viewertariat and BBC Question Time: Television Debate and Real-Time Commenting Online. The International Journal of Press/Politics, 16(4), 440-462. https://doi.org/10.1177/1940161211415519

Austin, J. L. (Ed.) (1962). How to do things with words. The William James Lectures delivered at Harvard University in 1955. Oxford University Press.

Barlovento Comunicación (23/04/2019). El Debate Electoral fue visto por 8,8 millones de espectadores [Press release]. https://www.barloventocomunicacion.es/audiencias-diarias/lunes-22$\underline{\text { abril-2019/ }}$

Benoit, W. L. (1999). Seeing spots: a functional analysis of presidential television advertisements, 1952-1996. Praeger.

Bentivegna, S. \& Marchetti, R. (2015). Live tweeting a political debate: The case of the 'Italia bene comune'. European Journal of Communication, 30(6), 631-647.

https://doi.org/10.1177/0267323115595526

Benveniste, É. (1958). De la subjetividad en el lenguaje. En Problemas de lingüística general. Volumen I. Siglo XXI.

Blas Arroyo, J. L. (1998). "Pero no me interrumpa usted, haga el favor" Las interrupciones en el debate político-electoral. Mediterranean Language Review, (10), 54-88.

Blas Arroyo, J. L. (1999). "Diga por qué, diga por qué...” La repetición en el debate político electoral. Revista de Investigación Lingüística, 2(1), 5-42.

Blas Arroyo, J. L. (2003). 'Perdóneme que se lo diga, pero vuelve usted a faltar a la verdad, señor Gonzalez': Form and function of politic verbal behaviour in face-to-face Spanish political debates. Discourse and Society, 14(4), 395-423. https://doi.org/10.1177/0957926503014004001 
Boyd, D., Golder, S. \& Lotan, G. (2010). Tweet, Tweet, Retweet: Conversational Aspects of Retweeting on Twitter. Comunicación presentada en la Hawaii International Conference on System Sciences-43, Hawaii. https://www.danah.org/papers/TweetTweetRetweet.pdf

Cabrejas-Peñuela, A. (2015). Manipulation in Spanish and American pre-election political debates:The Rajoy-Rubalcaba vs. Obama-McCain debates. Intercultural Pragmatics, 12(4), 515546. http://dx.doi.org/10.1515/ip-2015-0025

Cabrejas-Peñuelas, A. B. (2015). Manipulation in Spanish and American pre-election political debates: The Rajoy-Rubalcaba vs. Obama-McCain debates. Intercultural Pragmatics, 12(4), 515546. https://doi.org/10.1515/ip-2015-0025

Cabrejas-Peñuelas, A. B. \& Díez-Prados, M. (2014). Positive self-evaluation versus negative otherevaluation in the political genre of pre-election debates. Discourse and Society, 25(2), 159-185. https://doi.org/10.1177/0957926513515601

Callejón, P. L. (2001). La influencia de los debates electorales sobre la decisión de voto: el caso de mayo de 1993 en España. Revista Española de Ciencia Política, (5), 143-170.

Campos Freire, F. (2008). Las redes sociales trastocan los modelos de los medios de comunicación tradicionales. Revista Latina de Comunicación Social, (63), 277-286.

Cantavella-Blasco, J., Bullough, R., Curiel-Calleja, L. A., Blanco-Sieger, B. M., Mejía-Chiang, C. y Pittaro, E. (2008). Algunos aspectos lingüísticos de los debates electorales Zapatero-Rajoy 2008. Estudios sobre el Mensaje Periodístico, (14), 79-98.

Chadwick, A. (2011). Britain's First Live Televised Party Leaders' Debate: From the News Cycle to the Political Information Cycle. Parliamentary Affairs, 64(1), 24-44.

https://doi.org/10.1093/pa/gsq045

Chadwick, A., O’Loughlin, B. \& Vaccari, C. (2017). Why People Dual Screen Political Debates and Why It Matters for Democratic Engagement. Journal of Broadcasting \& Electronic Media, 61(2), 220-239. https://doi.org/10.1080/08838151.2017.1309415

Charaudeau, P. (2005). Quand l'argumentation n'est que visée persuasive. L'exemple du discours politique. En M. Burger y G. Martel. Argumentation et communication dans les médias. Éditions Nota Bene. https://www.patrick-charaudeau.com/IMG/pdf/2005_e_Argum-_et persuasion_Burger_pdf

Cortazar Rodríguez, F. J. (2014). Imágenes rumorales, memes y selfies: elementos comunes y significados. Iztapalapa, Revista de Ciencias Sociales y Humanidades, (77), 191-214.

Cuenca, M. J. y Marín, M. J. (2015). La representación discursiva del adversario en el debate electoral. Oralia, (18), 45-79.

del-Fresno García, M. y Daly, A. J. (2019). Límites para la comunicación política desde las grandes plataformas sociales de Internet. Un caso de estudio: de la casta a la trama. Revista Española de Investigaciones Sociológicas, (165), 65-82. https://doi.org/10.5477/cis/reis.165.65 
Doughty, M., Rowland, D. \& Lawson, S. (2012). Who is on Your Sofa? TV Audience Communities and Second Screening Social Networks. Comunicación presentada en el EuroITV 2012, Berlin.

Díez-Prados, M. \& Cabrejas-Peñuelas, A. B. (2018). Evaluation of "Status" as a Persuasive Tool in Spanish and American pre-electoral debates in times of Crisis. Atlantis, 40(2), 169-195. https://doi.org/10.28914/Atlantis-2018-40.2.09

El Heraldo (23/04/2019). Los mejores memes del debate en RTVE. https://www.heraldo.es/noticias/nacional/2019/04/22/debate-electoral-rtve-elecciones-generales2019-mejores-memes-redes-1310747.html

Elmer, G. (2012). Live research: Twittering an election debate. New Media \& Society, 15(1), 18-30. https://doi.org/10.1177/1461444812457328

FCINCO (23/04/2019). Los memes del debate electoral de TVE: propuestas para superar el tedio. El Mundo. https://www.elmundo.es/f5/2019/04/22/5cbe1de421efa08d2e8b45d6.html

Fernández García, F. (2009). Los debates Zapatero /vs/ Rajoy de 2008. Claves discursivas de una victoria. Linred: Lingüistica en la Red, (6), 1-37.

Fillmore, C. (1985). Frames and the semantics of understanding. Quaderni di Semantica, 6(2), 222254.

Fillmore, C. J. (1976). Frame Semantics and The Nature of Language. Annals of the New York Academy of Sciences, (280), 20-32.

Freelon, D. \& Karpf, D. (2015). Of big birds and bayonets: hybrid Twitter interactivity in the 2012 Presidential debates. Information, Communication \& Society, 18(4), 390-406. https://doi.org/10.1080/1369118X.2014.952659

G. C. (22/04/2019). Del 'marquito' de Rivera a los chopitos de Iglesias: las reacciones en las redes al debate. El Confidencial. https://www.elconfidencial.com/tecnologia/2019-04-22/memes-troleosbromas-debate-electoral-rtve 1956106/

Gallardo-Paúls, B. (1996). Análisis conversacional y pragmática del receptor. Episteme.

Gallardo-Paúls, B. (2013). Niveles pragmáticos y cognición: estrategias lingüísticas de encuadre en el discurso político. Anthropos, (239), 191-210.

Gallardo-Paúls, B. (2014). Usos políticos del lenguaje. Un discurso paradójico. Anthropos.

Gallardo-Paúls, B. (2017). Pseudopolítica en la red: indicadores discursivos de desideologización en Twitter. Pragmalingüística, (25), 8-30.

Gallardo-Paúls, B. (2018). Discurso político y desplazamientos discursivos. En C. E. Llamas (Ed.), El análisis del discurso político: géneros y metodologías. EUNSA.

Gallardo-Paúls, B. y Enguix Oliver, S. (2016). Pseudopolítica: el discurso político en las redes sociales. Valencia: Dept Teoria dels Llenguatges i Ciències de la Comunicació-UV. 
García-Marín, J. (2015). La cobertura mediática de los debates electorales en España Revista Española de Ciencia Política, (38), 135-161.

Gil de Zúñiga, H. \& Liu, J. H. (2017). Second Screening Politics in the Social Media Sphere: Advancing Research on Dual Screen Use in Political Communication with Evidence from 20 Countries. Journal of Broadcasting \& Electronic Media, 61(2), 193-219. https://doi.org/10.1080/08838151.2017.1309420

Guerrero-Solé, F. \& Mas-Manchón, L. (2017). Structure of the political tweets during the electoral campaigns of 2015 and 2016 in Spain. El Profesional de la Informacion, 26(5), 805-815.

Hosch-Dayican, B., Amrit, C., Aarts, K. \& Dassen, A. (2014). How Do Online Citizens Persuade Fellow Voters? Using Twitter During the 2012 Dutch Parliamentary Election Campaign. Social Science Computer Review, 34(2), 135-152. https://doi.org/10.1177/0894439314558200

Ibarretxe Antuñano, I. (2013). La lingüística cognitiva y su lugar en la historia de la lingüística. Revista española de lingüística aplicada, (26), 245-266.

Jefferson-Henrique (2016). Get Old Tweets Programmatically. Online: GitHub.

Justo, D. (23/04/2019). "En su cabeza sonaba espectacular": los memes sobre el primer debate electoral. cadenaser.com. https://cadenaser.com/ser/2019/04/23/politica/1555996283_414540.html

Kalsnes, B. (2014). Social media as a political backchannel. Aslib Journal of Information Management, 66(3), 313-328. https://doi.org/10.1108/AJIM-09-2013-0093

Kalsnes, B., Krumsvik, A. H. \& Storsul, T. (2014). Social media as a political backchannel. Aslib Journal of Information Management, 66(3), 313-328. https://doi.org/10.1108/AJIM-09-2013-0093

Lakoff, G. (1990). Women, fire, and dangerous things: what categories reveal about the mind. University of Chicago Press.

Lakoff, G. y Johnson, M. (1980). Metáforas de la vida cotidiana (C. González Marín, Trans.). Cátedra.

LOM. (23/04/2019). Los memes del debate de TVE. Levante-EMV. https://www.levanteemv.com/elecciones/2019/04/22/memes-debate-tve/1865516.html

López-García, G., Llorca-Abad, G., Valera-Ordaz, L. y Peris-Blanes, A. (2018). Los debates electorales, ¿el último reducto frente la mediatización? Un estudio de caso de las elecciones generales españolas de 2015. Palabra Clave, 21(3), 772-797.

https://doi.org/10.5294/pacla.2018.21.3.6

López-Meri, A. (2017). Contribución ciudadana al debate electoral y su cobertura periodística en Twitter. Prisma Social, (18), 1-33.

Mazzoleni, G. (2014). La comunicación política. Difusora Larousse - Alianza Editorial.

T. Meyer (2002). Media Democracy. How the Media Colonize Politics. Polity Press. 
Pedersen, S., Baxter, G., Burnett, S., Göker, A., Corney, D. \& Martin, C. (2015). Backchannel chat: peaks and troughs in a Twitter response to three televised debates during the 2014 Scottish Independence Referendum campaign, Krems, Austria.

Quintas Froufe, N. \& Quintas Froufe, E. (2010). Cara a cara electoral televisado: análisis audiovisual de los debates presidenciales. Miguel Hernández Communication Journal, (1), 20-39.

Redacción Yo Tele (23/04/2018). Los mejores memes del debate a cuatro organizado por RTVE. El Periódico de Cataluña. https://www.elperiodico.com/es/yotele/20190423/mejores-memes-debatecuatro-rtve-7419500

Roncallo-Dow, S., Córdoba-Hernández, A. M. \& Durán Camero, M. (2019). Aylan Kurdi, Twitter y la indignación efímera. Revista Española de Investigaciones Sociológicas, (165), 121-142. https://dx.doi.org/10.5477/cis/reis.165.121

Ruiz del Olmo, F. J. y Bustos Díaz, J. (2017). La evolución del debate televisivo como herramienta de comunicación política. Análisis del caso español: de la televisión a Twitter. Informação \& Sociedade: Estudos, 27(2), 235-252. https://doi.org/10.22478/ufpb.1809-4783.2017v27n2.32491

Rúas Araújo, J., Fernández Holgado, J. y Alén Amil, J. Á. (2018). La regulación de los debates electorales en el servicio audiovisual público. RISTI. Revista Ibérica de Sistema e Tecnologias de Informaçao, 16(11), 158-170.

Searle, J. R. (1969). Actos de habla: ensayo filosófico del lenguaje (1980 Ed.). Cátedra.

Shamma, D. A., Kennedy, L. \& Churchill, E. F. (2009). Tweet the debates: understanding community annotation of uncollected sources. Comunicación presentada en el Proceedings of the first SIGMM workshop on Social media, Beijing, China. https://doi.org/10.1145/1631144.1631148

Toffler, A. (1980). The Third Wave. William Morrow and Company.

Trilling, D. (2015). Two Different Debates? Investigating the Relationship Between a Political Debate on TV and Simultaneous Comments on Twitter. Social Science Computer Review, 33(3), 259-276. https://doi.org/10.1177/0894439314537886

Vaccari, C., Chadwick, A. \& O'Loughlin, B. (2015). Dual Screening the Political: Media Events, Social Media, and Citizen Engagement. Journal of Communication, 65(6), 1041-1061. https://doi.org/10.1111/jcom.12187

Vallespín, F. (2015). Política y nuevas redes. Revista TELOS. Revista de Pensamiento, Sociedad y Tecnologí, (100), 1-5.

van Dijk, T. A. (2003). Political Discourse and Ideology. Doxa Comunicación: revista interdisciplinar de estudios de Comunicación y Ciencias Sociales, (1), 207-222.

Vergeer, M. \& Franses, P. H. (2016). Live audience responses to live televised election debates: time series analysis of issue salience and party salience on audience behavior. Information Communication and Society, 19(10), 1390-1410. 
Verne. (23/04/2019). De las lecturas de la Constitución de Iglesias al silencio de Rivera: el debate en tuits. El País. https://verne.elpais.com/verne/2019/04/22/articulo/1555941993 271729.html

Villar-Hernández, P. (2019). Dime de qué hablas y te diré quién eres. El encuadre discursivo del periodista en Twitter durante la campaña electoral del 26J. In N. 1. Pellisser \& J. Oleaque (Eds.), Mutaciones discursivas en el siglo XXI: la politica en los medios y las redes. Tirant Humanidades.

Wells, C., Van Thomme, J., Maurer, P., Hanna, A., Pevehouse, J., Shah, D. V. \& Bucy, E. (2016). Coproduction or cooptation? Real-time spin and social media response during the 2012 French and US presidential debates. French Politics, 14(2), 206-233. https://doi.org/10.1057/fp.2016.4

Wodak, R. (2001). The discourse-historical approach. En R. Wodak \& M. Meyer (Eds.), Methods of Critical Discourse Analysis (pp. 63-94). SAGE Publications.

Zappavigna, M. (2011). Ambient affiliation: A linguistic perspective on Twitter. New Media \& Society, 13(5), 788-806. https://doi.org/10.1177/1461444810385097

\section{AUTORA:}

\section{Paz Villar-Hernández}

Doctora por la Universitat de València, Licenciada en Periodismo (Ciencias de la Información) por la Universitat Politècnica de València y Máster en Educación y TIC (Universitat Oberta de Catalunya). En la actualidad trabaja como técnico en el Servei de Formació Permanent i Innovació Educativa de la Universitat de València. Entre sus intereses de investigación están el discurso político, la comunicación audiovisual, los medios de comunicación, la edición científica y la Educación Superior. Es editora de la revista científica, Research in Education and Learning Innovation Archives (REALIA): https//www.uv.es/realia y forma parte de los Comités Editoriales de Journal of New Approaches of Educational Research (NAER) y RELIEVE (Revista Electrónica de Investigación y Evaluación Educativa) entre otros. Ha trabajado en Duke University (Estados Unidos) y ha realizado estancias en Northampton University y K.U. Leuven. Ha participado en los proyectos de investigación PRODISNET 01 y PRODISNET 02. (Diciembre 2019)

Paz.Villar@uv.es

Índice $\mathrm{H}: 2$

Orcid ID: http://orcid.org/0000-0001-9617-1688

Google Scholar: https://scholar.google.es/citations?user=RGGhsHgAAAAJ\&hl=es 